\title{
Learning and Remembering New Words: Clinical illustrations from Children with Specific \\ Language Impairment
}

Rouzana Komesidou, Department of Speech-Language-Hearing: Sciences and Disorders, University of Kansas, Lawrence, KS.

Holly L. Storkel, Department of Speech-Language-Hearing: Sciences and Disorders, University of Kansas, Lawrence, KS.

Disclosure: Rouzana Komesidou and Holly L. Storkel received salary support from the National Institute of Deafness and Other Communication Disorders (DC012824) to support completion of the research reported in this article. The authors have no nonfinancial relationship related to the content of this article.

\section{Introduction}

Learning new words has important implications for children's literacy development (Metsala \& Walley, 1998) and overall academic achievement. There is clear evidence that children with Specific Language Impairment (SLI) struggle with word learning (Dollaghan, 1987; Oetting, Rice, \& Swank, 1995; Kiernan \& Gray, 1998; McGregor, Newman, Reilly, \& Capone, 2002; Gray, 2003; 2004; Alt, Plante, \& Creusere, 2004; Alt \& Plante, 2006), suggesting the need for targeted interventions to support their success in the classroom. In the current article, we address the nature of word learning deficits in SLI from a neurocognitive perspective, and provide suggestions for treatment modifications to promote better learning outcomes by children with SLI.

Current theories of learning and memory differentiate at least two neurocognitive processes involved in word learning: 1) learning from input and 2) memory evolution in the absence of input (Davis, Di Betta, Macdonald, \& Gaskell, 2009; Davis \& Gaskell, 2009; 
McClelland, McNaughton, \& O’Reilly, 1995; Storkel, 2015). Learning from input occurs during the treatment session, when the clinician provides high-intensity and well-structured language input to the child. On the basis of this input, the child creates a new representation for a target word form and its object referent. For example, upon hearing the word "damp" the child will create a new representation of the word form (e.g., /dæmp/) and its object referent (e.g., a picture showing slightly wet clothes). Memory evolution refers to the learning that occurs outside the treatment session, and involves the integration of the new memory with existing memories based on linguistic (e.g., lexical, phonological, semantic) or property (e.g., functional, physical, categorical) similarities. For example, the word form representation of "damp" will be linked to the representation of existing words that are similar in form (e.g., "lamp", "dump"). Likewise, the semantic representation of "damp" will be linked to the representation of existing words that are similar in meaning (e.g., "wet", "moist"). The outcome of this integration can vary. On the one hand, integration may stabilize and strengthen the new memory leading to similar or better performance than when treatment ended. On the other hand, the newly created memory can be forgotten due to interference, decay, or competing memories (McGregor et al., 2013; Hardt, Nader, \& Nadel, 2013). It is important to note that forgetting could entail either the loss of some or all of the information in the newly created memory, or the loss of the ability to retrieve the newly created memory (Hardt et al., 2013).

Given this understanding of word learning as a two part neurocognitive process, it is possible that children with SLI could have limitations in either or both processes. Indeed, some evidence indicates difficulties with learning from input. Children with SLI have a poorer ability to associate a novel word with an unfamiliar object during minimal input than children with typical language (Gray 2003; 2004; Alt et al., 2004; Alt \& Plante, 2006). Poor word learning is 
also evident after additional exposures to the novel words (see Nash \& Donaldson, 2005).

However, children with SLI appear to benefit from increased input (Rice, Oetting, Marquis, Bode, \& Pae, 1994; Nash \& Donaldson, 2005), and in some cases, benefit more than typically developing peers (Riches, Tomasello, \& Conti-Ramsden, 2005). Additional evidence indicates that children with SLI have difficulties with memory evolution. Rice and colleagues (1994) found that word comprehension in children with SLI declined a few days after the last exposure. Similar findings were reported by Riches and colleagues (2005) for word production and comprehension. Thus, children with SLI may be more prone to forgetting than typically developing children when training is withdrawn. Taken together, children with SLI could struggle with learning from input and/or with memory evolution. The remainder of this article illustrates how a speech-language pathologist can use treatment data to identify whether a child is struggling with one or both of these processes and provides suggestions for modifying treatment to better facilitate word learning.

\section{Method}

\section{Interactive Book Reading}

Clinical illustrations were taken from an on-going preliminary clinical trial that uses interactive book reading to teach new words to kindergarten children with SLI (see Storkel, 2015; Voelmle \& Storkel, this issue). The clinical trial aims to identify an effective treatment intensity that will optimize learning outcomes for children with SLI. The clinical cases described here are from the intensity 36 condition. This intensity entails six exposures to target words within a book reading session and six repeated readings of each book over the course of the treatment (i.e., 6 exposures per book per session $\mathrm{x} 6$ sessions per book $=36$ total exposures by the end of treatment). A treatment session consists of previewing target words with a synonym 
(e.g., "Tailor is like sewer") and a definition (e.g., "Tailor means a person who makes or fixes clothes"), reading a given book and reintroducing the synonym when encountering the target word, and finally, reviewing target words using new pictures, context sentences (e.g., "The tailor fixed my dress that had a big hole.”), and previously introduced definitions. For a more detailed description of interactive book reading see Voelmle and Storkel in the current issue.

A naming task is administered periodically throughout the treatment to assess learning of treated and control words. It is important to note that learning of words is also assessed prior to and after treatment using a definition task (e.g., 'Tell me what tailor means'). Our existing data showed that children were not familiar with the treated words prior to treatment. During treatment, learning is monitored via a naming task because it is quicker to administer and score than the definition task. In the naming task, the experimenter shows one of the pictures used during the preview portion of the treatment and uses a question prompt such as "what are these?" or "how did he feel?" to elicit a naming response. For example, for the target word "snuggled", the child is shown a picture of an adult and a child lying together on the couch and asked "What did the people do?". The child must provide the relevant target word (snuggled), and his response is scored as either correct or incorrect. Note that correct responses include variations in tense (e.g., snuggled - snuggle/snuggling) and number (e.g., ripples - ripple). For intensity 36, learning during treatment was assessed after $6,12,24$, and 36 exposures. Data from the naming task are used to illustrate how a clinician can use treatment data to assess learning from input and memory evolution in the absence of input. Four different cases are used to illustrate different learning profiles. Although children generally learned the most words in the intensity 36 condition, individual differences in response to treatment were apparent. This variation makes it 
possible to identify different learning profiles that may require modification to the treatment for optimal outcomes.

\section{Learning Profiles}

We defined four learning profiles: 1) good learning from input and good retention, 2) poor learning from input but good retention, 3) good learning from input but poor retention, and 4) poor learning from input and poor retention. Tables 1, 2, 3, and 4 list the treated words that each child named correctly (out of a total of 30 words) at some point during treatment. The accuracy of those words is shown after $6,12,24$, and 36 exposures. At each exposure, the accuracy is displayed in the table as 0 -incorrect or 1-correct. The goal of reviewing these tables is to examine the pattern of correct and incorrect responses across exposures. For example, Table 1 shows that Child 015 named the target word "embarrassed" correctly for the first time after 6 exposures. Following 12 exposures, Child 015 named "embarrassed” correctly again, indicating that the child retained this word across exposures. Following 24 exposures, Child 015 named "embarrassed" incorrectly, indicating that the child may have temporarily forgotten the word. Finally, at 36 exposures (i.e., the last treatment session), Child 015 named "embarrassed" correctly again, indicating retention of the word at the conclusion of the treatment.

The bottom rows of each table summarize the overall pattern across words at each exposure in three ways: number of newly learned words, number of retained words across exposures, and total number of correct words. Newly learned words are defined as the number of words named correctly for the first time at a given test point. Retained words are defined as the number of words named correctly across test points (gray shading). The total number of correct words is the sum of newly learned words and retained words. Note that retention cannot be evaluated for words that are responded to correctly (i.e., newly learned words) at the last test 
point. For example, in Table 1, four words (squinting, glared, damp, discovered) are responded to correctly for the first time at the last test point so retention of these words cannot be judged because subsequent test points are not available.

Based on previous research (see Justice, Meier, \& Walpole, 2005), we defined the learning of a total of 4 or more words as a 'threshold response to treatment.' This criterion also was verified by examining accuracy for untreated words meeting the same criteria as the treated words. Here, children only provided correct responses to 0-3 untreated words with mean accuracy being less than 1 word (i.e., 0.83 ). Thus, learning 4 or more previously unknown words was viewed as being superior to no treatment. The average total number of correct words across children in intensity 36 was 6.5 (range 2-15). The average number of newly learned words was $2.2($ range $=2-15)$. The average percentage of words retained across children was $78 \%($ range $=$ $50 \%-100 \%$ ). Note that the percentage of words retained per child was calculated using the following formula: total number of retained words / (total number of correct words - newly learned words at last test point). These numbers served as the criteria to define good versus poor learning and good versus poor retention in the following profiles. That is, performance above the average level on total number of correct words, newly learned words, and percentage of words retained would indicate good learning from input and good retention. 
Table 1

Words learned by Child 015 following 6, 12, 24, and 36 exposures

\begin{tabular}{|l|c|c|c|c|}
\hline Correctly Named Words & $\mathbf{6}$ & $\mathbf{1 2}$ & $\mathbf{2 4}$ & $\mathbf{3 6}$ \\
\hline embarrassed & 1 & 1 & 0 & 1 \\
nervous & 1 & 0 & 1 & 1 \\
swung & 1 & 1 & 1 & 1 \\
hauled & 0 & 1 & 1 & 1 \\
hooves & 0 & 1 & 1 & 1 \\
silky & 0 & 1 & 0 & 0 \\
racket & 0 & 1 & 1 & 1 \\
swat & 0 & 0 & 1 & 1 \\
overjoyed & 0 & 0 & 1 & 1 \\
furnace & 0 & 0 & 1 & 1 \\
grumbling & 0 & 0 & 1 & 1 \\
prodded & 0 & 0 & 1 & 0 \\
sidelines & 0 & 0 & 1 & 1 \\
scarlet & 0 & 0 & 1 & 0 \\
squinting & 0 & 0 & 0 & 1 \\
glared & 0 & 0 & 0 & 1 \\
damp & 0 & 0 & 0 & 1 \\
discovered & 0 & 0 & 0 & 1 \\
\hline Newly Learned Words & 3 & 4 & 7 & 4 \\
Retained Words & - & 2 & 5 & 11 \\
\hline Total Correct Words & 3 & 6 & 12 & 15 \\
\hline Note. Dat from this hild & & 0 & \\
\hline
\end{tabular}

Note. Data from this child were also reported in Storkel (2015).

Profile 1: good learning from input and good retention. Child 015 acquired in total 15

of the 30 treated words over the course of his intervention, indicating robust learning. In terms of total words correct, this was the best performance among children in intensity 36 . This child also met the criterion for a minimal response to treatment (i.e., 4 words and above) and performed above average (i.e., 6.5) on total number of correct words. As shown in the bottom of Table 1, this child demonstrated good learning from input, with 3 to 7 newly learned words at each test point, which is above average (i.e., 2.2). In addition, this child showed strong retention. For example, the child learned 3 new words following 6 exposures and 2 of these words were named 
correctly at the following test point, 12 exposures. By the end of treatment, the child demonstrated retention of 11 out of the 15 learned words ( $79 \%$ of words were retained), which is just above average (i.e., 78\%). This pattern of words retained indicates good memory evolution in the absence of input. In general for this child, once a word was named correctly, the word tended to be named correctly throughout the remainder of treatment. The only exception to this pattern occurs for 3 words (silky, prodded, scarlet), for which correct responses were given at only one test point. It is possible that the child might have needed additional exposures or a different type of training to establish a strong representation for these specific items. However, this slight variability in responding to these 3 items is not a cause for concern, because overall a pattern of stability is demonstrated. As noted in the total correct words, good learning from input and good retention allowed the total number of correct words to increase across exposures. In this case, the total number increased from 3 words after 6 exposures to 15 words after 36 exposures. Overall, this particular case demonstrates the efficacy of shared book reading to increase vocabulary in children with SLI. This pattern of learning indicates that the treatment is working well for this child and modifications are not warranted.

Profile 2: poor learning from input but good retention. As shown in Table 2, Child 035 demonstrated relatively poor learning, acquiring only 4 of the 30 treated words by the end of treatment. Child 035 's overall learning appeared to be hampered by poor learning from input. Although Child 035 met our criterion for a minimal response to treatment (i.e., 4 or more total words correct), he performed below average (i.e., 6.5) on total number of correct words. This child acquired a first treated word (invisible) after 6 exposures, no new words after 12 exposures, a second treated word (snuggled) after 24 exposures, and finally two additional words (smooth, tight) after 36 exposures. Thus, the child learned only 0-2 words during input, which is below 
average (i.e., 2.2). Deficits in learning from input may be caused by deficits in procedural learning (Ullman \& Pierpont, 2005), affecting learning of word forms (see McGregor et al., 2013), or deficits in phonological working memory (Gathercole \& Baddeley, 1990), affecting learning of word forms and/or meaning. Relative to learning from input, however, retention was good for this child. The child retained correct responses to newly learned words (invisible, snuggled) across test points, demonstrating $100 \%$ retention, which is above average (i.e., $78 \%$ ). It is uncertain, however, whether similar retention trends would be observed for a larger number of words but first it is necessary to boost learning from input and then memory evolution could be evaluated more fully.

Table 2

Words learned by Child 035 following 6, 12, 24, and 36 exposures

\begin{tabular}{|l|c|c|c|c|}
\hline Correctly Named Words & $\mathbf{6}$ & $\mathbf{1 2}$ & $\mathbf{2 4}$ & $\mathbf{3 6}$ \\
\hline invisible & 1 & 1 & 1 & 1 \\
snuggled & 0 & 0 & 1 & 1 \\
smooth & 0 & 0 & 0 & 1 \\
tight & 0 & 0 & 0 & 1 \\
\hline Newly Learned Words & 1 & 0 & 1 & 2 \\
\hline Retained Words & - & 1 & 1 & 2 \\
\hline Total Correct Words & 1 & 1 & 2 & 4 \\
\hline
\end{tabular}

For this particular case, the clinician could consider increasing the number of exposures to target words during a treatment session, or include additional activities during the teaching episode to improve learning from input. Some examples of activities are having the child repeat the treated word (e.g., "say scarlet"), or guiding his attention to lexical (e.g., first sound, rhyme) and/or semantic (e.g., properties, features) characteristics of the new word. For example, upon presenting the word "scarlet" the clinician can say "Scarlet starts with an $s$ " or "Scarlet is a type of color". In addition, the clinician could have the child categorize the treated words (e.g., nouns 
versus verbs, words that refer to feelings). Finally, the clinician could include a recognition task, in which the child would identify treated word forms and referents from untreated word forms and referents. These modifications may help the child to use the input more efficiently and create a robust representation of the new word.

Profile 3: good learning from input but poor retention. As shown in Table 3, Child 022 demonstrated good, yet, modest learning, acquiring 8 of the 30 treated words by the end of treatment. Child 035 met the criterion for a minimal response to treatment (i.e., 4 or more total words correct) and his total words correct (i.e., 8) was above average (i.e., 6.5) but not as robust as Child 015 (i.e., 15). In terms of learning from input, Child 022 learned 3-5 new words at each test point which is above average (i.e., 2.2). Despite this relatively good learning from input, Child 022 demonstrated poor retention, with approximately $50 \%$ of the acquired words retained, which is below average (i.e., 78\%). Likewise, Child 022's word retention does not increase across exposures as observed for Child 015. Instead, Child 022's word retention remains relatively stagnant at 3-5 words at each test point. In addition, this child had a larger number of words $(n=6)$ than Child $015(n=3)$ that were named correctly at only one test point (flashing, gathered, awful, gulp, heaved, swaying). Taken together, Child 022's learning appears to be limited by memory evolution, such that previously learned words are forgotten across sessions. 
Table 3

Words learned by Child 022 following 6, 12, 24, and 36 exposures

\begin{tabular}{|l|c|c|c|c|}
\hline Correctly Named Words & $\mathbf{6}$ & $\mathbf{1 2}$ & $\mathbf{2 4}$ & $\mathbf{3 6}$ \\
\hline invisible & 1 & 1 & 1 & 1 \\
snuggled & 1 & 1 & 1 & 1 \\
tight & 1 & 1 & 1 & 1 \\
flashing & 1 & 0 & 0 & 0 \\
squawked & 0 & 1 & 1 & 0 \\
horrified & 0 & 1 & 0 & 1 \\
gathered & 0 & 1 & 0 & 0 \\
marvel & 0 & 0 & 1 & 1 \\
awful & 0 & 0 & 1 & 0 \\
gulp & 0 & 0 & 1 & 0 \\
heaved & 0 & 0 & 1 & 0 \\
swaying & 0 & 0 & 1 & 0 \\
haddock & 0 & 0 & 0 & 1 \\
swift & 0 & 0 & 0 & 1 \\
worn & 0 & 0 & 0 & 1 \\
\hline Newly Learned Words & 4 & 3 & 5 & 3 \\
\hline Retained Words & - & 3 & 4 & 5 \\
\hline Total Correct Words & 4 & 6 & 9 & 8 \\
\hline
\end{tabular}

Note. Data from this child were also reported in Storkel (2015).

Potential reasons for poor retention could be (1) rapid memory degradation before sleep related consolidation occurs, (2) competition between the new memory and old memories, or (3) difficulties with word retrieval. In terms of the first possibility, rapid memory degradation refers to the weakening of the initial representation of the word form and the object referent as the child engages in other tasks that delay sleep (see Storkel, 2005). To prevent rapid memory degradation, parents could practice the new words with their child before bed time, using the preview pictures and a script provided by the clinician. This might help the child to re-establish a correct word representation before sleep occurs. In addition, the clinician could consider increasing the number of treatment sessions or reducing the number of days between treatment sessions to minimize forgetting of newly acquired words. Turning to the second possibility, poor retention might occur due to competition between the new representation and similar existing 
representations. In this case, degradation of the new representation occurs when the new representation is linked with similar existing representations. This linking may lead to confusion between the new representation and old representations. To prevent interference from old memories, the clinician could help the child better differentiate old and new representations (e.g., bad vs. $a w f u l$ ), or include next day practice activities at home or in the classroom to allow relearning to occur. As a final possibility, poor retention could be also due to difficulties with word retrieval. Word retrieval can be facilitated with activities that include phonetic cues (e.g., the clinician can say the beginning sound or syllable to elicit the target word, use rhyming word cues), semantic cues (e.g., synonyms, antonyms, categories, attributes), sentence completion (e.g., the light was in the dark), and multiple-choice cueing (e.g., "is it invisible, flashing, or swaying?").

Profile 4: poor learning from input and poor retention. As shown in Table 4, Child 071 demonstrated overall poor word learning, with the total number of correct words at each test point ranging from 0 to 3 . This child did not meet our criterion for a minimal response to treatment (i.e., 4 or more total words correct) and performed below the average level (i.e., 6.5) on total number of correct words. This child exhibited challenges in both learning from input and memory evolution in the absence of input. The number of newly learned words remained low (i.e., 1- 2 words) across test points, and this was below average (i.e., 2.2). This pattern indicates poor learning from input. Child 071 also demonstrated poor retention of newly acquired words. Specifically, this child did not retain any newly learned words between 6 and 12 exposures, and retained 1 word between 12 and 24 exposures and 1 word between 24 and 36 exposures. This child's retention (i.e., 67\%) was below average (78\%). It is clear that this child requires modifications to enhance both learning from input and memory evolution. Consequently, 
changes should target treatment session activities to better support learning from input and between session activities to better support memory evolution. The discussion for Child 035 (e.g., increase the number of exposures, include lexical and semantic activities) provides guidance to clinicians on how the treatment session activities could be adjusted to accelerate learning from input. In addition, the discussion for Child 022 (e.g., include practice activities before and after sleep) provides suggestions on how between session activities could be adjusted to potentially improve memory evolution. Child 071 appears to need both types of modifications to successfully learn new words.

Table 4

Words learned by Child 071 following 6, 12, 24, and 36 exposures

\begin{tabular}{|l|c|c|c|c|}
\hline Correctly Named Words & $\mathbf{6}$ & $\mathbf{1 2}$ & $\mathbf{2 4}$ & $\mathbf{3 6}$ \\
\hline tight & 0 & 1 & 1 & 0 \\
pouted & 0 & 0 & 1 & 0 \\
swaying & 0 & 0 & 1 & 1 \\
snuggled & 0 & 0 & 0 & 1 \\
\hline Newly Learned Words & 0 & 1 & 2 & 1 \\
\hline Retained Words & - & 0 & 1 & 1 \\
\hline Total Correct Words & 0 & 1 & 3 & 2 \\
\hline
\end{tabular}

\section{Conclusion}

These clinical cases demonstrate how learning gains can vary between children with SLI receiving an interactive book reading treatment. Child 015 illustrated an optimal learning pattern characterized by good learning from input and good retention across sessions. A clinician who observes this type of pattern in treatment data can have confidence that the treatment is working effectively. In contrast, the other three children illustrated various departures from optimal learning, indicating that treatment modifications may need to be undertaken to maximize the effectiveness of the treatment. Depending on the pattern observed in the treatment data, treatment modifications will either target one (i.e., learning from input or memory evolution) or 
both neurocognitive processes. To optimize the effectiveness of interactive book reading for individual children, the clinician needs to closely monitor learning during the on-going treatment, determine whether the child demonstrates optimal learning from input and memory evolution, and consider alterations to the treatment in cases of weak learning from input and/or memory evolution.

\section{Acknowledgements}

The data described here are from an ongoing clinical trial supported by Grant DC 012824 from the National Institutes of Health (NIH). The contents are solely the responsibility of the authors and do not necessarily represent the official views of the NIH. We thank the children and their families who participated in this research. We also thank the speech-language pathologists, the school directors and the teachers for their involvement and support. Finally, we thank the staff of the KAW Story Project for their assistance with participant recruitment, data collection, and data processing. 


\section{References}

Alt, M., Plante, E., \& Creusere, M. (2004). Semantic features in fast-mapping performance of preschoolers with Specific Language Impairment versus preschoolers with normal language. Journal of Speech, Language, and Hearing Research, 47(2), 407-420.

Alt, M., \& Plante, E. (2006). Factors that influence lexical and semantic fast mapping of young children with specific language impairment. Journal of Speech, Language, and Hearing Research, 49(5), 941-954.

Davis, M. H., Di Betta, A. M., Macdonald, M. J., \& Gaskell, M. G. (2009). Learning and consolidation of novel spoken words. Journal of cognitive neuroscience, 21(4), 803-820.

Davis, M. H., \& Gaskell, M. G. (2009). A complementary systems account of word learning: neural and behavioural evidence. Philosophical Transactions of the Royal Society of London, Series B, Biological Sciences, 364(1536), 3773-3800.

Dollaghan, C. A. (1987). Fast mapping in normal and language-impaired children. Journal of Speech and Hearing Disorders, 52(3), 218-222.

Gathercole, S. E., \& Baddeley, A. D. (1990). The role of phonological memory in vocabulary acquisition: A study of young children learning new names. British Journal of Psychology, 81(4), 439-454.

Gray, S. (2003). Word-learning by preschoolers with Specific Language Impairment: What predicts success?. Journal of Speech, Language, and Hearing Research, 46(1), 56-67.

Gray, S. (2004). Word learning by preschoolers with Specific Language Impairment: Predictors and poor learners. Journal of Speech, Language, and Hearing Research, 47(5), 11171132. 
Hardt, O., Nader, K., \& Nadel, L. (2013). Decay happens: The role of active forgetting in memory. Trends in cognitive sciences, 17(3), 111-120.

Justice, L.M., Meier, J., \& Walpole, S. (2005). Learning new words from storybooks: An efficacy study with at-risk kindergartners. Language, Science, and Hearing Services in Schools, $36(1), 17-32$.

Kiernan, B., \& Gray, S. (1998). Word learning in a supported-learning context by preschool children with specific language impairment. Journal of Speech, Language, and Hearing Research, 41(1), 161-171.

McClelland, J. L., McNaughton, B. L., \& O'Reilly, R.C. (1995). Why there are complementary learning systems in the hippocampus and neocortex: Insights from the successes and failures of connectionist models of learning and memory. Psychological Review, 102(3), 419-457.

McGregor, K. K., Licandro, U., Arenas, R., Eden, N., Stiles, D., Bean, A., \& Walker, E. (2013). Why words are hard for adults with developmental language impairments. Journal of Speech, Language, and Hearing Research, 56(6), 1845-1856.

McGregor, K. K., Newman, R. M., Reilly, R. M., \& Capone, N. C. (2002). Semantic representation and naming in children with specific language impairment. Journal of Speech, Language, and Hearing Research, 45(5), 998-1014.

Metsala, J. L., \& Walley, A. C. (1998). Spoken vocabulary growth and the segmental restructuring of lexical representations: Precursors to phonemic awareness and early reading ability. In J. L. Metsala \& L. C. Ehri (Eds.), Word recognition in beginning literacy (pp. 89-120). Hillsdale, NJ: Erlbaum. 
Nash, M., \& Donaldson, M. L. (2005). Word learning in children with vocabulary deficits. Journal of Speech, Language, and Hearing Research, 48(2), 439-458.

Oetting, J. B., Rice, M. L., \& Swank, L. K. (1995). Quick incidental learning (QUIL) of words by school-age children with and without SLI. Journal of Speech, Language, and Hearing Research, 38(2), 434-445.

Rice, M. L., Oetting, J. B., Marquis, J., Bode, J., \& Pae, S. (1994). Frequency of input effects on word comprehension of children with specific language impairment. Journal of Speech, Language, and Hearing Research, 37(1), 106-122.

Riches, N. G., Tomasello, M., \& Conti-Ramsden, G. (2005). Verb learning in children with SLI: Frequency and spacing effects. Journal of Speech, Language, and Hearing Research, 48(6), 1397-1411.

Storkel, H. L. (2015). Learning from input and memory evolution: Points of vulnerability on a pathway to mastery in word learning. International Journal of Speech-Language Pathology, $17(1), 1-12$.

Ullman, M. T., \& Pierpont, E. I. (2005). Specific language impairment is not specific to language: The procedural deficit hypothesis. Cortex, 41(3), 399-433. 


\section{Learner objective:}

Identify strong versus weak word learning profiles in children with Specific Language Impairment (SLI) and modify treatment in cases of weak learning to promote better learning outcomes.

\section{Continuing education (CE) multiple choice questions:}

1. A child who learns 3-7 new words during each session and retains the majority of those words across sessions demonstrates:
A. Good learning from input and good memory evolution
B. Poor learning from input and good memory evolution
C. Good learning from input and poor memory evolution
D. Poor learning from input and poor memory evolution

Correct answer: A

Rationale: This example refers to Child 015 who showed good learning from input and good memory retention in the absence of input

2. A child who learns 0-2 new words during each session and retains the majority of those words across sessions demonstrates:
A. Good learning from input and good memory evolution
B. Poor learning from input and good memory evolution
C. Good learning from input and poor memory evolution
D. Poor learning from input and poor memory evolution

Correct answer: $B$

Rationale: This example refers to Child 035 who showed poor learning from input but good memory retention in the absence of input

3. A child who learns 3-5 new words during each session and retains approximately half of those words across sessions demonstrates:

A. Good learning from input and good memory evolution

B. Poor learning from input and good memory evolution

C. Good learning from input and poor memory evolution

D. Poor learning from input and poor memory evolution

Correct answer: $C$

Rationale: This example refers to Child 022 who showed good learning from input but poor memory retention in the absence of input

4. To improve learning from input for a child with SLI, the clinician could:

A. Increase the number of exposures to target words within a treatment session

B. Guide parents to practice new words with their child before and after sleep

C. Help the child differentiate new from old memories

D. Include word retrieval activities such as phonetic/semantic cueing or sentence completion

Correct answer: $A$

Rationale: $B, C$, and $D$ are used to improve memory retention 
5. To improve memory retention for a child with SLI, the clinician could:
A. Include a repetition task during the treatment session
B. Increase the number of treatment sessions and/or reduce the number of days between treatment sessions
C. Include a recognition task during the treatment session
D. Include a word categorization activity during the treatment session

Correct answer: $B$

Rationale: $A, C$, and $D$ are used to improve learning from input 